\title{
Connected majority domatic number of a graph
}

\author{
J. Joseline Manora ${ }^{1}$ and T. Muthukani Vairavel ${ }^{2 \star}$
}

\begin{abstract}
This paper introduces the concept connected majority domatic number of a graph $\mathrm{G}$, denoted as $d_{C M}(G)$. Also, exact values for some particular classes of graphs and bounds of $d_{C M}(G)$ are established.

Keywords

Connected Majority Dominating Set, Connected Majority Domination Number, Connected Majority Domatic Number.

AMS Subject Classification

05 C69.

${ }^{1}$ P.G and Research Department of Mathematics, TBML College, Porayar-609307, India.

${ }^{2}$ Department of Mathematics, Sir Issac Newton College, Nagapattinam-611002, India.

*Corresponding author: ${ }^{2}$ muthukanivairavel@gmail.com

Article History: Received 21 December 2018; Accepted 11 February 2019

(C)2019 MJM.
\end{abstract}

\section{Contents}

1

2

3

Introduction 52

Preliminaries .52

Connected Majority Domatic Number For Some Classes Of Graphs ........................ 53

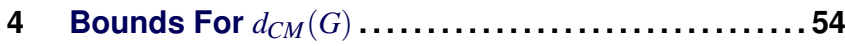

5 .55

\begin{abstract}
Rererences
\end{abstract}
\section{Introduction}

In this research article all graphs are simple, finite and undirected. The concept of domination arises naturally from location problems in operations research. The domination problem in a graph is to find a minimum sized vertex set $S$ such that every vertex not in $\mathrm{S}$ is adjacent to at least one vertex in S. Dominants and its variants have been extensively studied during the past decades. This paper considers a newly defined parameter namely connected majority domatic number of graph G. It is to find a maximum number of sets in the partition of $\mathrm{V}(\mathrm{G})$ into connected majority dominating sets of a graph G. For terminology and notation not specifically defined here, one can refer the reader[2].

A subset $S$ of $V(G)$ is said to be a dominating set [1],[2] of $G$ if every vertex in (V-S) is adjacent to at least one vertex in S. A dominating set is called minimal dominating set if no proper subset of $\mathrm{S}$ is a dominating set. The minimum cardinality of the minimal dominating set of $\mathrm{G}$ is called the domination number of $\mathrm{G}$, denoted by $\gamma(G)$. A domatic partition of a graph $G(V, E)$ is a partition of $V(G)$ all of whose classes are dominating sets of $\mathrm{G}$. The maximum number of classes of a domatic partition of $\mathrm{G}$ is called the domatic number of $\mathrm{G}$, denoted by $d(G)$.

A dominating set $\mathrm{S}$ is said to be a connected dominating set [2],[8] if the subgraph $\langle S\rangle$ induced by $\mathrm{S}$ is connected in G. The minimum cardinality of the minimal connected dominating set of $\mathrm{G}$ is called the connected domination number, denoted by $\gamma_{c}(G)$. A connected domatic partition of a graph $G(V, E)$ is a partition of $V(G)$ all of whose classes are connected dominating sets of $\mathrm{G}$. The maximum number of classes of connected domatic partition of $\mathrm{G}$ is called the connected domatic number[7] of $\mathrm{G}$, denoted by $d_{c}(G)$.

A subset $\mathrm{S}$ of $\mathrm{V}(\mathrm{G})$ is said to be a Majority Dominating set[4] if at least half of the vertices of $\mathrm{V}(\mathrm{G})$ are either in $\mathrm{S}$ or adjacent to elements of $\mathrm{S}$ i.e. $|N[S]| \geq\left\lceil\frac{V(G)}{2}\right\rceil$. A majority dominating set $\mathrm{S}$ is minimal if no proper subset of $\mathrm{S}$ is a majority dominating set. The minimum cardinality of a minimal majority dominating set is called Majority Domination number, denoted by $\gamma_{M}(G)$. A majority domatic partition of a graph $G(V, E)$ is a partition of $V(G)$ all of whose classes are majority dominating sets of $\mathrm{G}$. The maximum number of classes of majority domatic partition of $\mathrm{G}$ is called the Majority Domatic number[4] of G, denoted by $d_{M}(G)$.

\section{Preliminaries}

Definition 2.1. [3] A set $S \subseteq V(G)$ is a Connected Majority Dominating(CMD) set if 
i) $S$ is a majority dominating set and

ii) The subgraph $\langle S\rangle$ induced by $S$ is connected in $G$.

The connected majority dominating set $S$ is minimal if no proper subset of $S$ is a connected majority dominating set. The minimum cardinality of a minimal connected majority dominating set is called the Connected Majority Domination number and is denoted by $\gamma_{C M}(G)$.

Proposition 2.2. (i) For a path $P_{p}$ and a cycle $C_{p}, \gamma_{C M}\left(P_{p}\right)=$ $\gamma_{C M}\left(C_{p}\right)=\left\lceil\frac{p}{2}\right\rceil-2, p \geq 3$.

(ii) If the graphs $G=K_{p}, p \geq 2, K_{1, p-1}, p \geq 2, W_{p}, p \geq 5, F_{p}$, $p \geq 5, D_{r, s}, r, s \geq 2$, then $\gamma_{C M}(G)=1$.

Definition 2.3. The Connected Majority Domatic number $d_{C M}(G)$ of a graph $G$ is the maximum number of elements in a partition of $V(G)$ into connected majority dominating sets of $G$.

Definition 2.4. Consider the following graph $G$ with $p=15$.

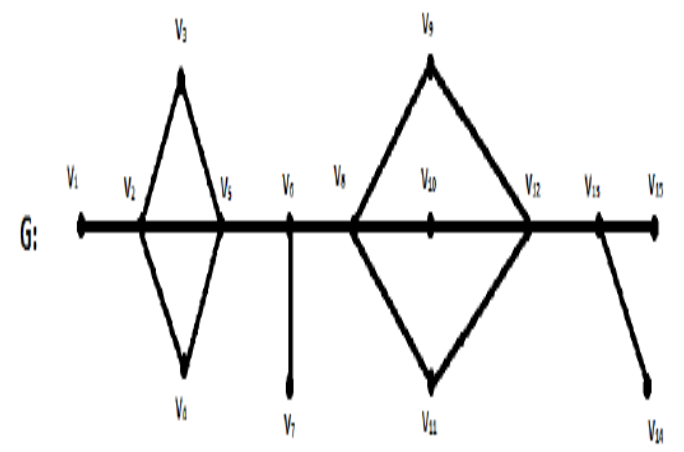

In $G, D_{1}=\left\{v_{2}, v_{5}, v_{6}\right\}, D_{2}=\left\{v_{6}, v_{8}, v_{10}\right\}, D_{3}=\left\{v_{8}, v_{10}, v_{12}\right.$, $\left.v_{13}\right\}, D_{4}=\left\{v_{15}, v_{13}, v_{12}, v_{10}\right\}$ are minimal connected majority dominating sets whereas $D_{4}=\left\{v_{1}, v_{2}, v_{3}, v_{4}, v_{5}, v_{6}\right\}$ is not a minimal connected majority dominating set. From these sets, $\gamma_{C M}(G)=3$. Again in $G$, the vertex set $V(G)$ is partitioned into connected majority dominating set of $G$ namely, $S_{1}=$ $\left\{v_{1}, v_{2}, v_{3}, v_{4}, v_{5}, v_{6}, v_{7}\right\}$ and $S_{2}=\left\{v_{8}, v_{9}, v_{10}, v_{11}, v_{12}, v_{13}, v_{14}\right.$, $\left.v_{15}\right\}$ are the only connected majority dominating sets of $G$,

$$
\therefore d_{C M}(G)=2 \text {. }
$$

\section{Connected Majority Domatic Number For Some Classes Of Graphs}

Proposition 3.1. For complete graph $K_{p}, K_{p}-e, K_{p}-2 e$, $d_{C M}\left(K_{p}\right)=p$

Proof. Since all vertices of these graphs are of degree $d(v) \geq$ $\left\lceil\frac{p}{2}\right\rceil-1$, each vertex is a connected majority dominating set of $\mathrm{G}$.

Proposition 3.2. For a Star $K_{1, p-1}, p \geq 5$, a subdivision $S\left(K_{1, p-1}\right)$ and $a$ wounded spider, $d_{C M}\left(K_{1 . p-1}\right)=1$.
Proof. Since all vertices $\mathrm{G}$ are having a full degree vertex and other vertices are pendants.

Proposition 3.3. For a Double Star $G=D(r, s), r \leq s$,

$d_{C M}(G)= \begin{cases}2, & \text { if } s=r, r+1 \\ 1, & \text { if } r<s\end{cases}$

Proposition 3.4. For any graph $G, d_{C M}(G) \leq\left\lfloor\frac{p}{\gamma_{C M}(G)}\right\rfloor$.

Proof. Let $\left\{V_{1}, V_{2} \ldots V_{k}\right\}$ be connected majority domatic partition of $V(G)$ and $d_{C M}(G)=k$.

Therefore, $V_{1} \cup V_{2} \ldots \cup V_{k}=V(G)$ and $p=\left|V_{1}\right|+\left|V_{2}\right|+\ldots+$ $\left|V_{k}\right| \geq k \cdot \gamma_{C M}(G)$. Then

$p \geq k, \gamma_{C M}(G)=d_{C M}(G), \gamma_{C M}(G)$.

Hence $d_{C M}(G) \leq\left\lfloor\frac{p}{\gamma_{C M}(G)}\right\rfloor$.

Proposition 3.5. For Path $G=P_{p}, p \geq 2$,

$d_{C M}\left(P_{p}\right)= \begin{cases}4, & \text { if } p=4,6 \\ 3, & \text { if } p=3,5,8 \\ 2, & \text { otherwise }\end{cases}$

Proof. Let $V\left(P_{p}\right)=\left\{u_{1}, u_{2}, \ldots u_{p}\right\}$ and $E\left(P_{p}\right)=\left\{u_{i}, u_{i+1} \mid 1 \leq\right.$ $i \leq p-1\}$.

$V\left(P_{p}\right)=\left\{u_{1}, u_{2}, \ldots u_{p}\right\}$ and $E\left(P_{p}\right)=\left\{u_{i}, u_{i+1} \mid 1 \leq i \leq p-1\right\}$. If $p=2$, then $d_{C M}(G)=2$.

Case (i): Let $p=4,6$.

Let $p=4$. Since each vertex of $P_{4}$ is a CMD set, $d_{C M}\left(P_{4}\right)=4$. Let $p=6$, Each internal vertex is the CMD set of $\mathrm{G}$, namely, are $S_{1}=\left\{v_{2}\right\}, S_{2}=\left\{v_{3}\right\}, S_{3}=\left\{v_{4}\right\}, S_{4}=\left\{v_{5}\right\}$. Therefore connected majority domatic partitions are $\left\{S_{1}, S_{2}, S_{3}, S_{4} \cup\right.$ $\left.\left\{v_{1}, v_{6},\right\}\right\}$. Hence, $d_{C M}\left(P_{6}\right)=4$.

Case (ii): Let $p=3,5,8$.

Let $p=3 \cdot d_{C M}(G)=3$.

Let $p=5$. Each internal vertex is a CMD set of $P_{5}$.

Therefore, $V\left(P_{5}\right)$ can be partitioned into three connected majority dominating sets of $\mathrm{G}$ namely, $\left\{S_{1}, S_{2}, S_{3} \cup\left\{v_{1}, v_{5}\right\}\right\}$. Let $p=8$. By Proposition 2.2, $\gamma_{C M}\left(P_{8}\right)=2$, the connected majority dominating sets of $\mathrm{G}$ are $S_{1}=\left\{v_{2}, v_{3}\right\}, S_{2}=\left\{v_{4}, v_{5}\right\}, S_{3}=$ $\left\{v_{6}, v_{7}\right\}$. Therefore $\left.V\left(P_{8}\right)\right)$ can be partitioned into only three CMD sets of G namely, $\left\{S_{1}, S_{2}, S_{3} \cup\left\{v_{1}, v_{8}\right\}\right\}$.

Hence, $d_{C M}\left(P_{8}\right)=3$.

Case (iii): Let $p \geq 9$ and $\mathrm{p}=7$.

Let $p=7$, By Proposition 2.2, $\gamma_{C M}\left(P_{7}\right)=2$. Then the vertex set is partitioned into $S_{1}=\left\{v_{2}, v_{3}\right\}, S_{2}=\left\{v_{4}, v_{5}\right\}$ and $R=\left\{v_{1}, v_{6}, v_{7}\right\}$ such that $\left|N\left[S_{i}\right]\right| \geq\left\lceil\frac{p}{2}\right\rceil$ and $\left\langle S_{i}\right\rangle$ is connected, $i=1,2$. It implies that $V(G)$ can be partitioned into $\left\{S_{1}, S_{2} \cup\right.$ $\left.\left\{v_{1}, v_{6}, v_{7}\right\}\right\}$, and the induced subgraph $\left\langle v_{1}, v_{6}, v_{7}\right\rangle$ is not connected. Hence, $d_{C M}\left(P_{7}\right)=2$.

Let $p \geq 9$. By Proposition 2.2, $\gamma_{C M}(G)=\left\lceil\frac{p}{2}\right\rceil-2$. Since for each connected majority dominating set $S$ of $G,\langle S\rangle$ is connected, $S_{1}=\left\{v_{2}, v_{3}, \ldots, v_{\left\lceil\frac{p}{2}\right\rceil-1}\right\}$ and

$S_{2}=\left\{v_{\left\lceil\frac{p}{2}\right\rceil}, v_{\left\lceil\frac{p}{2}\right\rceil+1}, \ldots, v_{\left\lceil\frac{p}{2}\right\rceil-1+\gamma_{C M}}\right\}$ are the only two connected majority dominating sets for $\mathrm{G}$. Then the remaining vertices $|R|=|V(G)|-\left|S_{1}+S_{2}\right|=p-2\left(\left\lceil\frac{p}{2}\right\rceil-2\right)$ and $|N[R]|<\left\lceil\frac{p}{2}\right\rceil$ and it would not be a connected majority dominating set of G. Hence, $S_{1}$ and $S_{2} \cup R$ are the connected 
majority domatic partition for $\mathrm{G}$. This implies that $d_{C M}(G) \geq$ 2. By proposition $3.4, d_{C M}(G) \leq\left\lfloor\frac{p}{\gamma_{C M}}\right\rfloor=\left\lfloor\frac{p}{\left\lceil\frac{p}{2}\right\rceil-2}\right\rfloor$, where $\gamma_{C M}(G)=\left\lceil\frac{p}{2}\right\rceil-2$. This implies that $d_{C M}(G) \leq 2$. Hence $d_{C M}(G)=2$, if $p \geq 9$.

Proposition 3.6. For Cycle $C_{p}, p \geq 3$

$d_{C M}\left(C_{p}\right)= \begin{cases}p, & \text { if } p=4,5,6 \\ 4, & \text { if } p=8 \\ 3, & \text { if } p=3,7,9,10 \\ 2, & \text { if } p \geq 11\end{cases}$

Proposition 3.7. For Wheel

$W_{p}=C_{p-1} \cup K_{1}, p \geq 5$

$d_{C M}\left(W_{p}\right)= \begin{cases}p, & \text { if } p=5,6,7,8 \\ 5, & \text { if } p=9,10 \\ 4, & \text { if } p=11,12,13,14,16 \\ 3, & \text { if } p=15 \text { and } p \geq 17\end{cases}$

Proof. Let $\left\{v_{1}, v_{2}, \ldots, v_{p}\right\}$ be the set of vertices of $G=W_{p}, p \geq$ 5. The vertex $\left\{v_{p}\right\}$ be the centre of $\mathrm{G}$ and the remaining vertices $\left\{v_{1}, v_{2}, \ldots, v_{p-1}\right\}$ form a outer cycle of a wheel graph $G=W_{p}$. Therefore $d\left(v_{p}\right)=p-1$ and $d\left(v_{i}\right)=3$, for $i=$ $1,2, \ldots, p-1$.

Case (i): Let $p=5,6,7,8$. Each vertex $\left\{v_{i}\right\}$ is a CMD set of G.

Case (ii): Let $p=9,10$.

When $p=9$. Let $S_{1}=\left\{v_{9}\right\}, S_{2}=\left\{v_{1}, v_{2}\right\}, S_{3}=\left\{v_{3}, v_{4}\right\}$,

$S_{4}=\left\{v_{5}, v_{6}\right\}, S_{5}=\left\{v_{7}, v_{8}\right\}$

When $p=10$. Let $S_{1}=\left\{v_{10}\right\}, S_{2}=\left\{v_{1}, v_{2}\right\}, S_{3}=\left\{v_{3}, v_{4}\right\}$,

$S_{4}=\left\{v_{5}, v_{6}\right\}, S_{5}=\left\{v_{7}, v_{8}\right\} \cup\left\{v_{9}\right\}$.

Therefore, $S_{1}, S_{2}, S_{3}, S_{4}$ and $S_{5}$ are the connected majority domatic partitions of $V(G)$. Hence, $d_{C M}(G)=5$ if $\mathrm{p}=9,10$.

Case (iii): Let $p=11,12,13,14,16$.

For $p=11$. Let $S_{1}=\left\{v_{11}\right\}, S_{2}=\left\{v_{1}, v_{2}, v_{3}\right\}, S_{3}=\left\{v_{4}, v_{5}, v_{6}\right\}$, $S_{4}=\left\{v_{7}, v_{8}, v_{9}\right\} \cup\left\{v_{10}\right\}$

For $p=12$. Let $S_{1}=\left\{v_{12}\right\}, S_{2}=\left\{v_{1}, v_{2}, v_{3}\right\}, S_{3}=\left\{v_{4}, v_{5}, v_{6}\right\}$, $S_{4}=\left\{v_{7}, v_{8}, v_{9}\right\} \cup\left\{v_{10}, v_{11}\right\}$

For $p=13$. Let $S_{1}=\left\{v_{13}\right\}, S_{2}=\left\{v_{1}, v_{2}, v_{3}, v_{4}\right\}, S_{3}=\left\{v_{5}, v_{6}\right.$, $\left.v_{7}, v_{8}\right\}, S_{4}=\left\{v_{9}, v_{10}, v_{11}, v_{12}\right\}$

For $p=14$. Let $S_{1}=\left\{v_{14}\right\}, S_{2}=\left\{v_{1}, v_{2}, v_{3}, v_{4}\right\}, S_{3}=\left\{v_{5}, v_{6}\right.$, $\left.v_{7}, v_{8}\right\}, S_{4}=\left\{v_{9}, v_{10}, v_{11}, v_{12}\right\} \cup\left\{v_{13}\right\}$

For $p=16$. Let $S_{1}=\left\{v_{16}\right\}, S_{2}=\left\{v_{1}, v_{2}, v_{3}, v_{4}, v_{5}\right\}, S_{3}=$ $\left\{v_{6}, v_{7}, v_{8}, v_{9}, v_{10}\right\}, S_{4}=\left\{v_{11}, v_{12}, v_{13}, v_{14}, v_{15}\right\}$.

For the above numbers, $\left|N\left[S_{k}\right]\right| \geq\left\lceil\frac{p}{2}\right\rceil$ and $d\left(v_{i}, v_{j}\right)=1 \forall v_{i}, v_{j} \in$ $S_{k}$ for $k=1,2,3,4$. Therefore $V(G)$ is partitioned into four connected majority dominating sets namely, $S_{1}, S_{2}, S_{3}$ and $S_{4}$. Hence $d_{C M}(G)=4$.

Case (iv): Let $p=15$ and $p \geq 17$.

Let $p=15$.

Let $S_{1}=\left\{v_{15}\right\}, S_{2}=\left\{v_{1}, v_{2}, v_{3}, v_{4}, v_{5}\right\}, S_{3}=\left\{v_{6}, v_{7}, v_{8}, v_{9}, v_{10}\right\}$, $R=\left\{v_{11}, v_{12}, v_{13}, v_{14}\right\}$ Such that $\left|N\left[S_{i}\right]\right| \geq\left\lceil\frac{p}{2}\right\rceil$ for $\mathrm{i}=1,2,3$. But $|N[R]|<\left\lceil\frac{p}{2}\right\rceil$. This implies that $V\left(W_{p}\right)$ can be partitioned into three connected majority dominating sets $\left\{S_{1}, S_{2}, S_{3} \cup R\right\}$. Hence, $d_{C M}\left(W_{p}\right)=3$.

Let $p \geq 17$.

Let $S_{1}=\left\{v_{p}\right\}, S_{2}=\left\{v_{1}, v_{2}, \ldots, v_{\left\lceil\frac{p}{2}\right\rceil-3}\right\}$ and $S_{2}=\left\{v_{\left\lceil\frac{p}{2}\right\rceil-2}\right.$, $\left.v_{\left\lceil\frac{p}{2}\right\rceil-1}, \ldots v_{2\left(\left\lceil\frac{p}{2}\right\rceil-3\right)}\right\} S_{3}=\left\{v_{\left\lceil\frac{p}{2}\right\rceil-2}, v_{\left\lceil\frac{p}{2}\right\rceil-1}, \ldots v_{2\left(\left\lceil\frac{p}{2}\right\rceil-3\right)}\right\}$ be the three sets with $d\left(v_{i}, v_{j}\right)=1$, for all $v_{i}, v_{j} \in S_{k}$ for $\mathrm{k}=2,3$ and $i \neq j$ and the remaining vertices $R=\left\{v_{2\left\lceil\frac{p}{2}\right\rceil-5}, v_{2\left\lceil\frac{p}{2}\right\rceil-4}, \ldots\right.$, $\left.v_{p-1}\right\}$. Since $\left\{v_{p}\right\}$ is a full degree vertex, $\left|N\left[S_{1}\right]\right|>\left\lceil\frac{p}{2}\right\rceil$. Since $S_{2}, S_{3} \subseteq V\left(C_{p-1}\right)$ and $\left|N\left[S_{2}\right]\right|=\left\lceil\frac{p}{2}\right\rceil-3+3=\left\lceil\frac{p}{2}\right\rceil$ and $\left|N\left[S_{3}\right]\right|=$ $2\left(\left\lceil\frac{p}{2}\right\rceil-3\right)-\left(\left\lceil\frac{p}{2}\right\rceil-3\right)+3=\left\lceil\frac{p}{2}\right\rceil$. This implies that $S_{k}, k=$ $1,2,3$ are all CMD sets of $\mathrm{G}$. But we obtain the vertices

$R=\left\{v_{2\left\lceil\frac{p}{2}\right\rceil-5}, v_{2\left\lceil\frac{p}{2}\right\rceil-4}, \ldots, v_{p-1}\right\}$, each vertex with $d\left(v_{i}, v_{j}\right)=$ 1 , for all $v_{i}, v_{j} \in R$

$|N[R]|=(p-1)-\left(2\left\lceil\frac{p}{2}\right\rceil-4\right)+3<\left\lceil\frac{p}{2}\right\rceil$. Therefore $\mathrm{R}$ is not a CMD set of $\mathrm{G}$ always. Hence, the vertex set of $\mathrm{G}$ can be partitioned into 3 CMD sets $\left\{S_{1}, S_{2}, S_{3} \cup R\right\}$. Hence, $d_{C M}\left(W_{p}\right)=$ 3.

Proposition 3.8. Let $K_{m, n}, m \leq n$ be a complete bipartite graph with $m+n=p$. Then

$d_{C M}(G)= \begin{cases}p, & \text { if } n=m, m+1, m+2 \\ m, & \text { if } m<n\end{cases}$

Proof. Let $V_{1}(G)=\left\{u_{1}, u_{2} \ldots u_{m}\right\}$ and $V_{2}(G)=\left\{v_{1}, v_{2} \ldots v_{n}\right\}$ be bipartitions of $\mathrm{V}(\mathrm{G})$.

Case (i): Let $n=m, m+1, m+2$. Then $\left|N\left[u_{i}\right]\right| \geq\left\lceil\frac{p}{2}\right\rceil$ and $\left|N\left[v_{i}\right]\right|=\left\lceil\frac{p}{2}\right\rceil$. Therefore each

$\left\{u_{i}\right\}, i=1,2, \ldots m$ and $\left\{v_{i}\right\}, i=1,2, \ldots n\left\{v_{j}\right\}, j=1,2, \ldots n$ are CMD sets of $\mathrm{G}$. Hence

$d_{C M}(G)=m+n=p$ if $n=m, m+1, m+2$.

Case (ii): Let $m<n$. Then $n \geq m+3$.

In $V_{1}(G)$, each vertex $u_{i}$ of degree $d\left(u_{i}\right)=n$ and in $V_{2}(G)$, each vertex $v_{i}$ of degree $d\left(v_{i}\right)=m$.

If $p=\left|V_{1}\right|+\left|V_{2}\right|=n+m$, then $\left\lceil\frac{p}{2}\right\rceil=\left\lceil\frac{m+3+m}{2}\right\rceil=\left\lceil\frac{2 m+3}{2}\right\rceil=$ $m+2$, when $\mathrm{n}=\mathrm{m}+3$. Therefore, each $u_{i}$ covers at least $\left|N\left[u_{i}\right]\right|=n+1=m+4$, vertices, when $\mathrm{n}=\mathrm{m}+3$.

Since every vertex $\left\langle u_{i}\right\rangle$ is connected and $\left|N\left[u_{i}\right]\right|>\left\lceil\frac{p}{2}\right\rceil$, each $\left\{u_{i}\right\}, i=1,2, \ldots m$ in $V_{1}(G)$ is a CMD set for $\mathrm{G}$. The vertices in $V_{2}(G)$ form a majority dominating set for $\mathrm{G}$ but the induced graph could not be connected. So, the vertices of $V_{2}(G)$ could not form a connected majority dominating set for $\mathrm{G}$. Hence, $d_{C M}(G)=m$ if $n \geq m+3$ and $m<n$.

\section{Bounds For $d_{C M}(G)$}

Theorem 4.1. For any graph $G$ with $p$ vertices, $d_{C M}(G)=p$ if and only if $\delta(G) \geq\left\lceil\frac{p}{2}\right\rceil-1$.

Proof. Let, $d_{C M}(G)=p$. Then every vertex $u \in V(G)$ forms a connected majority dominating set for G. Thus $d(u) \geq\left\lceil\frac{p}{2}\right\rceil-$ 1 , for every $u \in V(G)$. Hence $\delta(G) \geq\left\lceil\frac{p}{2}\right\rceil-1$. Conversely, suppose $\delta(G) \geq\left\lceil\frac{p}{2}\right\rceil-1$. Then $d(u)=\delta(G)$, for any $u \in$ $V(G)$ and $d(v) \geq \delta(G) \geq\left\lceil\frac{p}{2}\right\rceil-1, d(u)=\delta(G)$, for any $u \in$ $V(G)$ and $d(v) \geq \delta(G) \geq\left\lceil\frac{p}{2}\right\rceil-1$, for every $v \in V(G)$. It implies that each vertex $v$ of $\mathrm{G}$ is a CMD set of $\mathrm{G}$.

Hence $d_{C M}(G)=p$.

Result 4.2. Given any positive integer $k$, there exists a graph $G$ which is not complete for which $d_{C M}(G)=k$. 
Proof. Let $G=K_{\left\lfloor\frac{k}{2}\right\rfloor,\left\lceil\frac{k}{2}\right\rceil}$. Since each vertex of G is a connected majority dominating set, $d_{C M}(G)=k$.

Proposition 4.3. Let $T$ be a tree with $p \geq 3$ and $e>2$, pendants. If $\operatorname{diam}(T)=2$ or 3 , then $d_{C M}(T)=1$ or 2 .

Proof. Let $\operatorname{diam}(T)=2$. Then the tree $T=K_{1, p-1}$ and $\gamma_{C M}(T)=1$. Therefore let $S=\{u\}$, where $u$ is the central vertex of T. Since all other vertices are pendants, $\langle V-\{u\}\rangle$ could not form a CMD set for G. Hence, $d_{C M}(G)=1$.

Let $\operatorname{diam}(T)=3$. Then $T$ is a double star. Let $x$ and $y$ be the centers of $T$ and $\left\{u_{1}, u_{2}, \ldots, u_{m}\right\}$ be the set of pendants adjacent to $x$ and $\left\{v_{1}, v_{2}, \ldots, v_{n}\right\}$ be the set of pendants adjacent to $y$. Let $|V(T)|=m+n+2$.

Case (i): Let $m<n$ and $n \geq m+3$ is even.

Let $S_{1}=\{y\}$ and $S_{2}=\{x\}$. Then $\left|N\left[S_{1}\right]\right| \geq\left\lceil\frac{p}{2}\right\rceil$ and $\left|N\left[S_{2}\right]\right|<$ $\left\lceil\frac{p}{2}\right\rceil$. Here $S_{1}$ is the only CMD set of $\mathrm{G}$ and $S_{2}$ could not be a connected majority dominating set of $\mathrm{G}$. Also, all pendant sets $\left\{u_{i}\right\}$ and $\left\{v_{i}\right\}$ could not form a connected majority dominating set of $\mathrm{G}$. It implies that there is only one connected majority domatic set in $V(G)$. Hence $d_{C M}(T)=1$.

Case (ii): Let $n=m, m+1, m+2$.

The only connected majority domatic partitions of $V(T)$ are the centres of T i.e., $S_{1}=\{x\}$ and $S_{2}=\{y\}$ such that $\left|N\left[S_{1}\right]\right| \geq$ $\left\lceil\frac{p}{2}\right\rceil$ and $\left|N\left[S_{2}\right]\right| \geq\left\lceil\frac{p}{2}\right\rceil$. This implies that $d_{C M}(T)=2$.

Proposition 4.4. If $G$ is a complete graph then $d_{C M}(G)=$ $d_{M}(G)=d_{C}(G)$.

Proof. Since every vertex of a complete graph $\mathrm{G}$ is a full degree vertex, each vertex is a connected dominating set or majority dominating set or connected majority dominating set of G. Hence $d_{C M}(G)=d_{M}(G)=d_{C}(G)=p$ if $G=K_{p}$.

Proposition 4.5. If a graph $G$ has exactly one full degree and others are pendant vertices then $d_{C M}(G)=d_{C}(G)$.

Corollary 4.6. For a subdivision graph $S\left(K_{1, p-1}\right)$ with each edge is divided by any number of times, then $d_{C M}(G)=$ $d_{C}(G)=1$.

Proof. Since the full degree vertex v of $\mathrm{G}$ is a connected dominating set and also connected majority dominating set of $\mathrm{G}$, the remaining all vertices could not form a connected dominating set or connected majority dominating set for $\mathrm{G}$. Therefore, the vertex set $\mathrm{V}(\mathrm{G})$ cannot be partitioned into either connected dominating set or connected majority dominating set of $\mathrm{G}$. Hence, $d_{C M}(G)=d_{C}(G)=1$.

Proposition 4.7. For any graph $G$,

(i). $d_{C M}(G) \leq d_{M}(G)$ and

(ii). $d_{C M}(G) \geq d_{C}(G)$ (i) $d_{C M}(G) \geq d_{M}(G)$ and (ii) $d_{C M}(G) \geq$ $d_{C}(G)$.

Proof. (i).Since every connected majority dominating set of $\mathrm{G}$ is a Majority Dominating set of $\mathrm{G}, \gamma_{C M}(G) \geq \gamma_{M}(G)$. Hence, $d_{C M}(G) \leq d_{M}(G)$. (ii) Since every Connected Dominating set of $\mathrm{G}$ is a Connected Majority Dominating set of $\mathrm{G}, \gamma_{C}(G) \geq \gamma_{C M}(G)$. Hence, $d_{C}(G) \leq d_{C M}(G)$.

Proposition 4.8. For any graph $G$,

$d_{C}(G) \leq d_{C M}(G) \leq d_{M}(G)$.

Proof. It follows from Proposition 4.7.

Proposition 4.9. Let $G$ be any 3-regular graph with ' $p$ ' vertices. Then

$$
d_{C M}(G)= \begin{cases}p, & \text { if } p=4,6,8 \\ 5, & \text { if } p=12 \\ 4, & \text { if } p=10 \text { and } p \geq 14 .\end{cases}
$$

Proof. Let $V(G)=\left\{v_{1}, \ldots v_{p}\right\}$ be the set of 'p' vertices in $\mathrm{G}$. Case (i) : Let $p=4,6,8$. Since each vertex $\left\{v_{i}\right\}$ is a CMD set of $\mathrm{G}, d_{C M}(G)=p$.

Case (ii): Let $p=12$. Since $\gamma_{C M}(G)=\left\lfloor\frac{p-1}{4}\right\rfloor$, consider $S_{1}=\left\{v_{1}, v_{6}\right\}, S_{2}=\left\{v_{2}, v_{7}\right\}, S_{3}=\left\{v_{3}, v_{8}\right\}, S_{4}=\left\{v_{4}, v_{9}\right\}$,

$S_{5}=\left\{v_{5}, v_{10}\right\}$ with $d\left(v_{i}, v_{j}\right)=1$, for every $v_{i}, v_{j} \in S_{k}, k=$ $1, \ldots, 5$ and $i \neq j$. Then $\left|N\left[S_{k}\right]\right| \geq\left\lceil\frac{p}{2}\right\rceil$, for every $k=1, \ldots, 5$. Therefore the sets $S_{k}$ are the only CMD sets of G. In this structure, the remaining vertices $R=\left\{v_{11}, v_{12}\right\}$ with distance $d\left(v_{11}, v_{12}\right)=2$, which is not connected. Therefore, there are $\left\{S_{1}, S_{2}, S_{3}, S_{4}\right.$

$\left.S_{5} \cup R\right\}$ are the five connected majority domatic partitions of G. Hence, $d_{C M}(G)=5$.

Case (iii): (a) Let $p=10$. Since $\gamma_{C M}(G)=\left\lfloor\frac{p-1}{4}\right\rfloor$, consider $S_{1}=\left\{v_{1}, v_{5}\right\}, S_{2}=\left\{v_{2}, v_{6}\right\}, S_{3}=\left\{v_{3}, v_{7}\right\}, S_{4}=\left\{v_{4}, v_{8}\right\}$ with $d\left(v_{i}, v_{j}\right)=1$, for every $v_{i}, v_{j} \in S_{k}, k=1, \ldots, 4$ and $i \neq j$. Then $\left|N\left[S_{k}\right]\right| \geq\left\lceil\frac{p}{2}\right\rceil$, for every $k=1, \ldots, 4$. Then the sets $S_{k}$ are the only CMD sets of $\mathrm{G}$. In this structure, the remaining vertices $R=\left\{v_{9}, v_{10}\right\}$ with distance $d\left(v_{9}, v_{10}\right) \geq 2$, which is not connected. Therefore, there are $\left\{S_{1}, S_{2}, S_{3}, S_{4} \cup R\right\}$ are the four connected majority domatic partitions of $\mathrm{G}$. Hence, $d_{C M}(G)=4$.

Case (iii): (b) Let $P \geq 14$. In the structure, $v_{1}$ is adjacent to three vertices namely, $v_{\frac{p}{2}}, v_{\frac{p}{2}+1}, v_{\frac{p}{2}+2}$. Similarly, $v_{2}$ is adjacent to three vertices $v_{\frac{p}{2}+1}, v_{\frac{p}{2}+2}, v_{\frac{p}{2}+3}$. Similarly, $v_{3}$ is adjacent to three vertices $v_{\frac{p}{2}+2}, v_{\frac{p}{2}+3}, v_{\frac{p}{2}+4}$ and so on. $S_{1}=$ $\left\{v_{1}, v_{\frac{p}{2}}, v_{p-1}, v_{\frac{p}{2}-2, \ldots}\right\}, S_{2}=\left\{v_{2}, v_{\frac{p}{2}+1}, v_{p}, v_{\frac{p}{2}-1, \ldots}\right\}$, $S_{3}=\left\{v_{3}, v_{\frac{p}{2}+4}, v_{5}, v_{\frac{p}{2}+6, \ldots}\right\}, S_{4}=\left\{v_{4}, v_{\frac{p}{2}+5}, v_{6}, v_{\frac{p}{2}+7, \ldots}\right\}$ such that $d\left(v_{i}, v_{j}\right)=1$, for every $v_{i}, v_{j} \in S_{k}, k=1, \ldots, 4$ and $i \neq j$ and $\left|N\left[S_{k}\right]\right| \geq\left\lceil\frac{p}{2}\right\rceil$. Therefore $S_{k}$ are the only CMD sets of $\mathrm{G}$ and the remaining vertices form the set $R=V(G)-\bigcup_{i=1}^{4} S_{i}$ at a distance 2 which is not connected. Therefore,there are $\left\{S_{1}, S_{2}, S_{3}, S_{4} \cup R\right\}$ are the four connected majority domatic partitions of $\mathrm{G}$. Hence, $d_{C M}(G)=4$.

\section{Conclusion}

In this article, researcher introduced and discussed new type of parameter of a graph G. Connected majority domatic number of a graph is defined and it is determined for some 
classes of graphs. This concept is useful in communication network and in many real-life situations. Also, this concept can be extended to product graphs.

\section{References}

[1] Cockayane E.J. and Hedetniemi S.T., Towards a theory of domination in graphs, Networks. 7(1977), 247-261.

[2] Haynes T.W, Hedetniemi S.T and Slater P.J, Fundamentals of domination in graphs, Marces Dekker. Inc, New york, 1998.

[3] Joseline Manora J and Muthukani Vairavel T, Connected Majority Dominating Set of a Graph, Global Journal of Pure and Applied Mathematics, 13(2)(2017), 534-543.

[4] Joseline Manora J and Swaminathan V, Majority Domatic number of a Graph, Global Journal of Pure and Applied Mathematics, 6(3)(2010), 275-283.

[5] Joseline Manora J and Swaminathan V, Majority Dominating sets, JARJ, 3(2)(2006), 75-82.

[6] Joseline Manora J and Swaminathan V, Results on Majority Dominating sets, Scientia Magna, 7(3)(2011), 53-58.

[7] Paulraj Joseph J and Arumugam S, On the connected domatic number of a graph, J. Ramanujam Math. Soc., 9(1)(1994), 69-77.

[8] Sampathkumar E and Walikar H.B, The connected domination number of a graph, J. Math. Phy. Sci., 13(6)(1979), $12-19$.

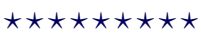

$\operatorname{ISSN}(\mathrm{P}): 2319-3786$

Malaya Journal of Matematik

ISSN(O):2321 - 5666

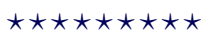

